\title{
Revelation of Self-organizing Theory on the Construction of E-Training Systems
}

\author{
Xin Huang \\ Tsinghua University, Beijing, China
}

\begin{abstract}
A good quality e-training system could provide powerful support and assurance to the development of etraining. From the perspective of self-organizing theory, etraining with its appearing characteristics of openness, nonequilibrium, non-linear, and random fluctuation provides revelation to the construction of e-training system. Namely, the construction of e-training system should provide sufficient approaches to acquire rich resources, support different learning patterns to facilitate autonomous learning, construct individualized learning environment, and implement training management system based on self-awareness and selffulfillment.
\end{abstract}

\section{INTRODUCTION}

With the rapid development of information technologies, e-training has become one of the principal ways to achieve individual continuous education and lifelong learning. E-training could not develop without the support of its system that not only provides the environment on the implementation of it, but also has direct impact on its effects. Nowadays, there are many studies on e-training systems that are however just based on the construction and application in fundamental level, such as the construction of an e-training system for different learners in a particular field with different training requirements, but lack of the research and design in overall level.

Self-organization is an essential attribute of all systems and there is no system existing without Self-organization [1]. Analyzing the construction of e-training system from the perspective of self-organizing theory can make the research and design of the system more efficient and in turn better serve learners. As a carrier to implement e-training, e-training system can achieve individualization, intellectualization, and provide better services to the learners by following the characteristics and rules of selforganizing theory. Self-organizing theory provides the guidance to the construction of e-training system and brings revelations to the process. This study will firstly analyze the problems that current e-training systems have, and then summarize the principle characteristics of the theory and finally provide suggestions to the construction of e-training system.

\section{INADEQUATE OF E-TRAINING SYSTEM}

Nowadays, e-training has been widely used in various industries. Although some achievements have been made, there are also some shortcomings:

\section{A. Single Method to Acquire Training Resources}

The prominent problem of e-training is that learners often rely on experts or other external knowledge. The results of which led directly to learner is only ready to accept learning contents in a passive way. In fact, the process of training is a self-growth process, rather than passive learning process. E-training learners have the specific characteristics of adult learners. They may be experts in particular fields. They can be both recipients and providers of the knowledge. They often have many theories and practical experiences to share with others[2].

\section{B. Monotonous Learning Pattern for Learners}

Traditional training emphasizes on knowledge transfer to learners. Compared with traditional training, e-training attaches great importance to develop learners' abilities in many aspects. However, e-training is still mainly on the knowledge transfer, which makes learning pattern dull. In web 2.0, it should be highlighted in learner-centered models. Thus, different learning patterns should be provided, depending on the type of learning contents, learner's characteristics, etc. Those learning patterns include the research-based teaching, problem-based learning, and project-based learning etc.

\section{Inadequate Support for Personalized Learning Environment}

In big data era, it is claimed to make higher demands on personalized learning environment for learners, which is helpful to keep the continuous and persistent learning. With the increasing demands on e-training, many e-training systems are emergent. However, these systems could only provide simple techniques such as statistical analysis, to support personalized learning. As a result, some of them are insufficient in supporting personalized services.

\section{Lack of Emotional Experience}

Traditional e-training focuses on the knowledge transmission, but may lack of the emotional experiences of the learners. In fact, it is inherent intertwined in emotion and cognition. Knowledge is with emotion as the premise, and cognition is based on emotional preference[3]. Knowledge is formed through interaction, communication, consultation and consensus, rather than by giving the rigid knowledge. Thus, e-training is not just to advance training skills or solve problems, but should focus on interaction among learners and teachers, and achieve a variety of interactive ways by technologies to support emotional communication. 


\section{SElF-ORganizing CHARACTERISTICS Of E- TRAINING SYSTEM}

\section{A. Self-Organizing Theory}

Self-organization is the process in which system evolution is not driven by external forces, and each part in the system is coordinated in each other, resulting in orderly structures[4]. Self-organizing theory reveals system evolution in natural view. It points out that evolution of a system is not affected by external intervention, but by the interaction between each component in the system, which facilitate the emergence of the ordered structures. A selforganizing system is determined by the interaction among multiple factors. It mainly includes[5]: (1) Only the characteristic of openness can make the system become ordered from disordered; (2) Far from equilibrium is the condition necessary for the system to become selforganizing; (3) Nonlinear interaction between elements could produce synergistic effect. (4) Random fluctuation may greatly interfere with or promote the learning process, so as to obtain different results.

\section{B. Self-Organizing Characteristics of E-Training}

E-training consists of multiple elements, such as teachers, learners, techniques, learning contents, teaching methods, activities, learning environment, etc. In traditional training, teachers play a leading role, and learners passively receive learning contents. It is a form of self-organizing process. But it is often regulated by human resources management from institutions. According to this, it shows a behavior like "other-organization". Along with the status and role of the market in resource allocation increasing prominently and the change of social demands increasing rapidly, planning and scheduling learning materials in the systematic way has been unable to adapt to the changing demands in the market. E-training is an useful way for learners to get knowledge actively, which could make them adopt themselves to the changing market rapidly. E-training gradually turns to "self-organization" process. Additionally, the characteristics of openness, non-equilibrium, non-linear and fluctuation show that e-training has the self-organizing characteristics, as is depicted in figure 1.

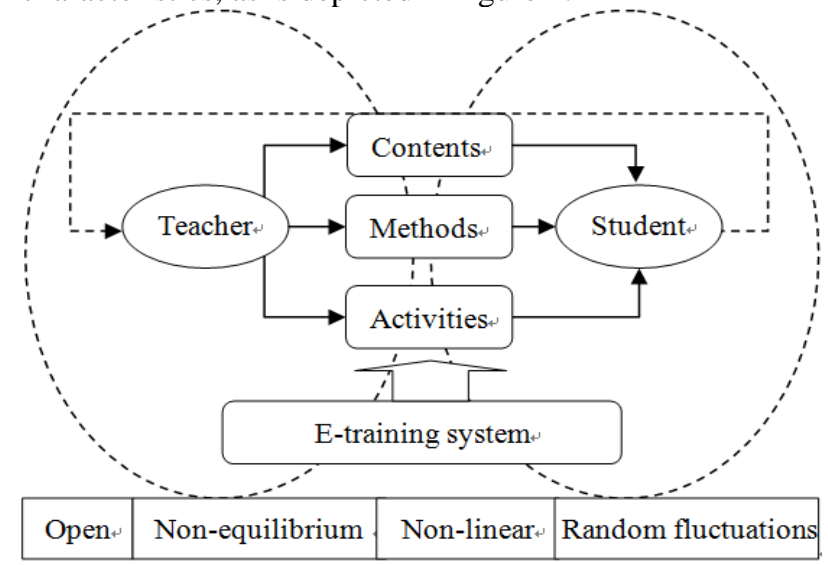

Figure 1. Self-organization characteristics of e-training system

\section{1) Openness}

The openness characteristic of e-training is to ensure the learning process through the exchange of matters, energies and information from outside. E-training is interacted with the external environment, including society, economy and culture, etc, rather than existed in isolation. Specific performances include: regardless of learning time and space, diversified modes for acquiring knowledge, open learning contents and learning ways, etc.

\section{2) Non-linear}

In traditional training, structured and systematic learning contents are transferred to the learners by teachers according to certain teaching steps at a fixed time and place. E-training reflects the non-linear characteristic in learning process. Sometimes, learners have different educational backgrounds, learning motivations and learning styles. Also, unstructured contents, random learning processes and diversified training outputs fully reflect the non-linear characteristic of e-training, making e-training more vitality. The open environment of e-training allows learners to be independent, autonomous, self-planning and self-management. Autonomy is one of the distinctive features of non-linear.

\section{3) Non-equilibrium}

Non-equilibrium is the source of orderliness. In modern times, four major challenges are globalization, high-quality, diversity and high-performance, which make the end of lifelong employment, but the coming era of lifelong learning. With the knowledge freshness getting more and more shorter, the demands for e-training have become more and more urgent. Learners constantly interacted with external environment lead to the needs of learning and the appearance of non-equilibrium in self-organizing activities. Non-equilibrium drives learners constantly adapt to environmental changes.

\section{4) Random fluctuations}

In the process of e-training, learning contents, learning methods, learning time, learning interests and other factors are interrelated. Slight fluctuation of an element may be amplified by non-linear mechanism and transited to a new stable and orderly situation. In the process of autonomous learning, information is to be enlarged, causing fluctuation and unexpected results, and then enters into a new advanced state.

\section{Revelations Of Self-Organizing Theory To THE CONSTRUCTION OF E-TRAINING SYSTEM}

The following part will analyze the self-organizing theory to the construction of e-training system and reveal the guidance and revelation to the construction process.

\section{A. Provide Ways to Acquire Rich Resources Based on The Openness Characteristic}

According to the theory, with the openness and interactive communication of an e-training system, teachers and learners could construct and assess resources, which in turn make the resources present the trend of self-increasing. 
The characteristics of openness and non-linear have appeared here. In self-organizing resources model, due to the openness characteristic of the e-training system, interactions among resources, teachers and learners provide useful review and feedback in the process of organizing resources. Therefore, the process can be optimized and selforganizing can be achieved[6]. Self-organizing cannot work well without the role of interaction. It is necessary to have multiple means to support interactions so that learners can choose interactive tools based on their own needs. The etraining system should provide all kinds of real time or non real time interactive tools, such as discussion forum, message center, email, text message, online Q\&A, learning community and etc. Multiple interactive tools can extend the accessibility for obtaining knowledge.

\section{B. Support Different Learning Models Aimed at Autonomous Learning}

According to the theory's characteristics of openness and non-equilibrium, the construction of e-training system should support different learning models, such as resourcebased learning, community-based learning and coursebased learning, etc. The learning process of e-training is an autonomous-learning process. It emphasizes the learners' self-organizing process in which learners transform themselves from random learning to self-organizing learning, from passive learning to active learning, and from non- persistent learning to persistent learning[7]. Those three transforming processes indicate the self-organizing process and the progressive learning process of learners from low lever to high level.

\section{Construct Individualized Training Environment Based on Big Data Technologies}

According to the theory's non-equilibrium and random fluctuation characteristics, big data analysis technologies should be taken to construct the individualized learning environment for learners. The e-training system accumulates a large number of fine-grained data about learners' learning behaviors, such as learning logs, learning path, learning outcomes data, course data, learning management data, etc [8]. Useful information could be minded and analyzed by utilizing data mining technologies, providing powerful technologies for the construction of individualized learning environment.

\section{Implement Training Management System Based on Self- Awareness and Self-Fulfillment Process Technologies}

The theory emphasizes learners' autonomous and personalized learning ways. Thus, the construction of etraining system should take full advantage of processoriented management techniques to provide the dynamic management of self-awareness and self-fulfillment processes. E-portfolio[9] is as an efficient tool for selfmanagement and self-reflection, which encourages selforganizing abilities of learners by providing individualized e-portfolios. By capturing the detail information, such as interactive communication, self-planning, self-reflection, working experience and etc, it may improve their selfawareness and self-fulfillment so that better self-organizing can be achieved.

\section{CONCLUSIONS}

This paper presents the construction of e-training system based on self-organizing theory. Self-organization is the nature of complex system. By analyzing the selforganizing characteristics of e-training, it is beneficial to improve the rationality of constructing e-training system. Our study may be as a reference for further research and development of e-training.

\section{REFERENCES}

[1] S. Wang, "Discuss the self-organizing mechanism of e-learning system", Journal of southwest agricultural university(social science edition), 2006(3), pp.273-276.

[2] M. S. Knowles. The making of an adult educator: An autobiographical journey. San Francisco: Jossey-Bass, 1989.

[3] A. Hargreaves, "The emotional practice of teaching", Teaching and Teacher Education, 1998(4), pp.835-854.

[4] Science of System Dictionary, Editor Committee. Science of System Dictionary, Kunming: Yunnan Technology Publish, 1983.

[5] H. Haken, "Information and self-organization: A macroscopic approach to complex systems", Berlin \& New York: Oxford University press Inc,1998.

[6] W.H. Ma, Y.H. Liu, "Study on Self-Organizing Construction of Network Teaching Resources", Journal of Modern Information, 2011(6), pp.166-170.

[7] X.X. Jia, "Research on the inquiry-based Learning and receptive learning from self-organizing theory's perspective", Journal of Teaching and Management, 2009(6), pp.16-17.

[8] P. Xu, Y.N. Wang, Y.H. Liu, H. Zhang, "The Learning Innovation from the Perspective of Big Data: An Analysis of the U.S. Report of Enhancing Teaching and Learning through Educational Data Mining and Learning Analytics and Its Enlightenment", Journal of distance education, 2013(6), pp.11-17.

[9] I. Balaban, E. Mu, B. Divjak, "Development of an electronic Portfolio system success model: An information systems approach", Computers \& Education, 2013(1), pp.396-411. 